\title{
Elimination of cervical cancer as a public health problem-how shorter brachytherapy could make a difference during COVID-19
}

\author{
Aparna Gangopadhyay \\ Independent Practice, 377, M. B. Road, Panchanantala, Kolkata 700049, India
}

\section{Abstract}

The World Health Organization has called for elimination of cervical cancer as a public health problem and has adopted strategies in this regard. However, the estimates for achieving the goals depend on the ability to provide timely treatment in a certain proportion of cases. The coronavirus disease 2019 pandemic has had a serious impact on healthcare delivery in many low and middle income countries (LMICs) with the highest burden of cervical cancer; funds and infrastructure are being reallocated to deal with the emergency, and cancer care has been seriously affected. In the absence of clear and reliable estimates, the exact extent of disruption remains unclear. It is, therefore, essential that pragmatic approaches are adopted to save lives. There has been considerable debate regarding the use of the $9 \mathrm{~Gy} \times 2$ fractions high dose rate brachytherapy schedule for the treatment of locally advanced cervical carcinoma. However, in LMICs with the highest global burden of locally advanced cervical cancer cases, radiation facilities have been using this fractionation schedule in many cases to deal with the overwhelming number of patients, who would have otherwise been denied timely treatment. In view of the current pandemic, and the difficulties in accessing and delivering timely healthcare, mortality owing to delayed treatment cannot be denied in LMICs, which already have underequipped healthcare facilities. Use of the shortest available fractionation schedule to provide timely treatment would serve to save more lives in regions with high incidence and mortality from the disease.

Keywords: brachytherapy, cervix uteri, COVID-19 pandemic, radiation dose fractionation

\section{The cervical cancer problem in low and middle income countries}

Low and middle income countries (LMICs) are the major contributors to the global burden of cervical carcinoma, with most cases presenting at locally advanced stages; these countries also report the highest mortality rates from the disease [1]. The numerous challenges to the prevention, diagnosis and treatment of cervical carcinoma in these settings have considerable impact on the number of completed treatments and lives saved [2]. Along with the availability of other treatment modalities, radiotherapy capacity is limited owing to the lack of infrastructure, medical personnel and representative data [3]. In addition, repeated hospital visits are considerably inconvenient for most patients owing
Correspondence to: Aparna Gangopadhyay Email: mails7778@gmail.com

ecancer 2022, 16:1352

https://doi.org/10.3332/ecancer.2022.1352

Published: 07/02/2022

Received: 30/09/2021

Publication costs for this article were supported by ecancer (UK Charity number 1176307).

Copyright: (c) the authors; licensee ecancermedicalscience. This is an Open Access article distributed under the terms of the Creative Commons Attribution License (http:// creativecommons.org/licenses/by/4.0), which permits unrestricted use, distribution, and reproduction in any medium, provided the original work is properly cited. 
to difficulties in transportation and socioeconomic reasons [4, 5]. Moreover, high-quality health care is inequitably distributed in many LMICs, and socioeconomically disadvantaged and vulnerable patients often receive poor quality care, in terms of both competence of healthcare personnel and treatment experience [6].

\section{Issues with brachytherapy}

Brachytherapy has been an integral component of radiotherapy for carcinoma of the cervix, as it improves radiocurability by allowing the delivery of high doses of radiation to the central tumour volume [7]. Although traditionally delivered by low dose rate equipment, the use of high dose rate (HDR) treatment delivery has become popular worldwide owing to the possibility of outpatient treatment, avoidance of radiation exposure to staff, consistent and reproducible applicator positioning and dose optimisation, achieved with a variable dwell-time stepping source [8]. In LMICs, there are substantial disparities between radiotherapy capacity and patient requirements. Brachytherapy services are difficult to access and are mostly located in larger urban centres. Patient waiting times are therefore long, making timely delivery of treatment an area of considerable concern. Owing to the greater possibility of normal tissue sparing with advancements in technology, it has been possible to use larger fraction sizes, which allows earlier treatment completion [9]. In low resource regions, where patient turnover rates and treatment compliance are major issues, hypofractionation is widely employed for increasing patient turnover and treatment compliance with encouraging results $[10,11]$.

Hypofractionated schedules serve to reduce the number of visits and increase patient turnover rates, both of which are particularly necessary in these settings. In view of the current coronavirus disease 2019 (COVID-19) pandemic, there have been difficulties in healthcare delivery worldwide $[12,13]$. In particular, the already overburdened radiation therapy facilities in LMICs have been further affected by reallocation of funds and shortage of available staff $[14,15]$. The use of hypofractionated schedules has, therefore, become particularly relevant in this scenario.

\section{Guidelines for dose-fractionation of HDR brachytherapy for cervical cancer}

The American Brachytherapy Society (ABS) consensus guidelines for HDR brachytherapy in locally advanced cervical carcinoma recommend the administration of five or six fractions, delivered weekly [16]. A report by the ABS Task Group also suggests the use of smaller fraction sizes for reducing treatment toxicity [17]. However, a multi-institutional trial that compared the outcomes between HDR brachytherapy delivered in four 7-Gy and two 9-Gy fractions demonstrated similar overall survival and a comparable incidence of serious toxicities [18].

\section{Clinical use of the $9 \mathrm{~Gy} \times 2$ regimen}

The LMICs in Southeast Asia remain the major contributors to the global burden of cervical carcinoma and also have the highest mortality rates from the disease [1]. In view of the limited infrastructure, shortage of trained staff and patient convenience, there has always been a compelling need to implement clinically feasible shorter fractionation schedules for HDR brachytherapy. As the shortest fractionation schedule, the $9 \mathrm{~Gy} \times 2$ regimen has, therefore, gained particular popularity in these regions, and numerous studies have demonstrated the safety and efficacy of this regimen [9-11]. In this context, it is noteworthy that many brachytherapy facilities in LMICs lack facilities for interstitial needle placement, solely depending on the use of intracavitary applications. In locally advanced cases, tumour regression following external beam radiotherapy is often delayed owing to the considerable burden of parametrial disease; clinicians in underequipped facilities, therefore, often wait for optimal tumour regression to ensure adequate coverage of the clinical target volume. In such cases, the 2 -fraction regimen provides a pragmatic means of completing definitive treatment on schedule. However, the proposed recommendations published in view of the COVID-19 pandemic have advised against the use of the 9 Gy $\times 2$ regimen, citing its radiobiological disadvantages [19]. 


\section{Impact of treatment on cervical cancer-related mortality in LMICs}

The overall incidence of cancer is lower in LMICs compared to that of high-income countries; however, total cancer-related mortality is significantly higher in LMICs, especially among those younger than 65 years. The significant economic impact resulting from premature mortality and lost years of productivity is an area of considerable concern [20]. The United Nations sustainable development goals intend to achieve a one-third reduction in premature mortality from non-communicable diseases by 2030 [21]. As cervical cancer is both preventable and curable, the World Health Assembly passed a resolution in August 2020, calling for the elimination of cervical cancer as a public health problem, and also adopted a strategy to realise this goal [22]. This is first ever initiative for the elimination of a cancer worldwide.

The World Health Organization (WHO) has accordingly developed the WHO Cervical Cancer Elimination Modelling Consortium, involving three independent dynamic models of human papilloma virus infection, cervical carcinogenesis, screening and precancer and invasive cancer treatment. The triple-intervention strategy adopted by the WHO involves the use of vaccination strategies, screening and treatment for eliminating cervical cancer. It is expected that this strategy will prevent approximately a third of the premature deaths from cervical cancer in LMICs over the next 10 years, and nearly $90 \%$ of deaths over the next century.

A recent study estimated the reductions in age-standardised rates of cervical cancer mortality in 78 low-income and lower-middle-income countries for three core scenarios, namely, girls-only vaccination; girls-only vaccination with once-lifetime screening and cancer treatment scale-up; and girls-only vaccination with twice-lifetime screening and cancer treatment scale-up [23]. The study reported that the estimated mortality rate due to cervical cancer across all the 78 LMICs was 13.2 per 100,000 women in 2020. The findings suggested that compared to the status quo, vaccination alone would have minimal impact on cervical cancer mortality by 2030 , with an estimated reduction of $0.1 \%$. However, additional scaling up of twice-lifetime screening and cancer treatment would reduce mortality by $34.2 \%$, thereby preventing an estimated 300,000 deaths by 2030 . It has been projected that twice-lifetime screening and cancer treatment would prevent $14 \cdot 6$ million deaths by 2070 , and $62 \cdot 6$ million deaths by 2120 .

The study concluded that the strategy may achieve a one-third reduction in the rate of premature mortality from cervical cancer in LMICs over the next 10 years, and will thus allow realisation of the 2030 United Nations sustainable development goals. In addition, the successful implementation of the elimination strategy proposed by the WHO would reduce mortality due to cervical cancer by nearly $99 \%$, and save more than 62 million lives over the next century.

\section{Concerns in view of the COVID-19 pandemic}

Notably, the mentioned study assumed that half of the patients with invasive cervical cancer would receive adequate treatment by 2023; this was expected to increase to $90 \%$ by 2030 [23]. However, there are no current estimates on the number of patients with cervical cancer who are being denied or are unable to access screening and treatment services during the COVID-19 pandemic. Nevertheless, recent reports from the Asia-Pacific region suggest that similar to other regions across the globe, cancer care is being considerably affected by the pandemic [24-26], with screening and treatment services being equally affected [27, 28]. A surge in cases has, therefore, been expected on resumption of usual services [29, 30]. In LMICs, where cancer care facilities are already overburdened, treatment delays are inevitable. Patient turnover, therefore, assumes particular importance in the successful reduction of mortality.

In the context of cervical cancer, most patients present in locally advanced stages in these settings [1]. The standard treatment, therefore, essentially comprises whole pelvic external beam radiation and concurrent chemotherapy [31], with brachytherapy as an integral component. In view of the paucity of brachytherapy facilities available in these settings, and the reallocation of funding during the pandemic, access to brachytherapy has been particularly restricted [32, 33].

Hypofractionation, therefore, represents a feasible approach for improving patient turnover. As the shortest available HDR fractionation schedule for locally advanced cervical cancer, the $9 \mathrm{~Gy} \times 2$ fractions regimen gains particular importance in saving lives.

A multicentre international clinical trial that recruited 601 patients, demonstrated no significant differences in terms of overall survival or severe treatment-related side effects between patients who received HDR brachytherapy with four fractions of 7 Gy and those who received two fractions of 9 Gy each [18]. 
In view of the prevailing difficulties in treatment during the pandemic, it is clear that the reduction of even a single extra fraction may offer definite benefits in terms of patient turnover in the overburdened brachytherapy facilities of LMICs [34].

\section{Case of a major global contributor: India}

India is one of the major contributors to the global mortality from cervical cancer, accounting for nearly one-third of cervical cancer deaths worldwide. Women in this vast nation have a 1.6\% cumulative risk of developing cervical cancer and a $1.0 \%$ cumulative risk of death from the disease [35]. A report on cancer statistics from the National Cancer Registry Programme in India indicated that most cervical cancer cases continue to present at locally advanced stages in the country, and that chemoradiation remains the commonest treatment administered [36]. In this context, it is worth noting that many cases are missed during data collection, as cancer is not a notifiable disease in India; actual incidence and mortality figures are therefore likely to be higher. Estimates from a study in India suggested that delays in the diagnosis and treatment due to the COVID-19 pandemic will increase the number of deaths from cervical cancer [37].

\section{Why a shorter brachytherapy schedule may make a difference}

LMICs face serious constraints in radiotherapy capacity, delaying timely intervention in many cases. Brachytherapy is particularly resource intensive, and there are numerous financial and accessibility issues that hinder access to timely treatment in these settings. Use of the shorter 2 -fraction regimen will reduce patient waiting times and increase the number of completed treatments within a given timeframe; this, in turn will translate to more lives being saved. In the face of overwhelming patient numbers, the shorter schedule will also improve treatment quality in the overburdened radiotherapy facilities of LMICs.

\section{Conclusions}

Although overburdened brachytherapy facilities in LMICs have been using shorter fractionation schedules to ensure timely delivery of treatment to many patients with cervical cancer, the need for expediting treatment completion has never been as critical as in the era of COVID19. The serious impact on screening and treatment accessibility implies that the number of locally advanced cases is set to rise in future; increased cervical cancer related mortality is therefore inevitable. Under the circumstances, shorter brachytherapy fractionation will serve as a notable life-saver in the difficult times of COVID-19 and offer a practical means for realizing the United Nations sustainable development goals.

\section{Funding}

None.

\section{Conflicts of interest}

None.

\section{Acknowledgments}

The author would like to thank Dr Aditi Gangopadhyay, Postdoctoral Research Associate, University of Calcutta for assistance with manuscript preparation. 


\section{References}

1. Sung H, Ferlay J, and Siegel RL, et al (2021) Global cancer statistics 2020: GLOBOCAN estimates of incidence and mortality worldwide for 36 cancers in 185 countries CA Cancer J Clin 71 209-249 https://doi.org/10.3322/caac.21660 PMID: 33538338

2. Randall TC and Ghebre R (2016) Challenges in prevention and care delivery for women with cervical cancer in Sub-Saharan Africa Front Oncol 28160

3. Grover S, Xu MJ, and Yeager A, et al (2015) A systematic review of radiotherapy capacity in low- and middle-income countries Front Oncol 4380 https://doi.org/10.3389/fonc.2014.00380 PMID: 25657930 PMCID: 4302829

4. Syed ST, Gerber BS, and Sharp LK (2013) Traveling towards disease: transportation barriers to health care access J Community Health 38 976-993 https://doi.org/10.1007/s10900-013-9681-1 PMID: 23543372 PMCID: 4265215

5. Mattson J (2011) Transportation, distance, and health care utilization for older adults in rural and small urban areas Trans Res Rec 2265 192-199 https://doi.org/10.3141/2265-22

6. Kruk ME, Gage AD, and Arsenault C, et al (2018) High-quality health systems in the Sustainable Development Goals era: time for a revolution Lancet Glob Health 6 e1196-e1252 https://doi.org/10.1016/S2214-109X(18)30386-3 PMID: 30196093 PMCID: 7734391

7. Haie-Meder C, Pötter R, and Van Limbergen E, et al (2005) Gynaecological (GYN) GEC-ESTRO Working Group. Recommendations from Gynaecological (GYN) GEC-ESTRO Working Group (I): concepts and terms in 3D image based 3D treatment planning in cervix cancer brachytherapy with emphasis on MRI assessment of GTV and CTV Radiother Oncol74 235-245 https://doi.org/10.1016/j. radonc.2004.12.015 PMID: 15763303

8. Stewart AJ and Viswanathan AN (2006) Current controversies in high-dose-rate versus low-dose-rate brachytherapy for cervical cancer Cancer 107 908-915 https://doi.org/10.1002/cncr.22054 PMID: 16874815

9. Sood BM, Gorla G, and Gupta S, et al (2002) Two fractions of high-dose-rate brachytherapy in the management of cervix cancer: clinical experience with and without chemotherapy Int J Radiat Oncol Biol Phys 53 702-706 https://doi.org/10.1016/S0360-3016(02)02745-1 PMID: 12062615

10. Thakur P, Dogra E, and Gupta M, et al (2019) Comparison of iso-effective and cost-effective high-dose-rate brachytherapy treatment schedules in cervical cancer - regional cancer center experience J Contemp Brachytherapy 11 428-435 https://doi.org/10.5114/ jcb.2019.88329 PMID: 31749851 PMCID: $\underline{654857}$

11. Ghosh S, Rao PB, and Kotne S (2015) High dose rate brachytherapy in two 9 Gy fractions in the treatment of locally advanced cervical cancer-a South Indian institutional experience Asian Pac J Cancer Prev 16 7167-7170 https://doi.org/10.7314/APJCP.2015.16.16.7167

12. Jazieh AR, Akbulut $H$, and Curigliano G, et al (2020) International research network on COVID-19 impact on cancer care. Impact of the COVID-19 pandemic on cancer care: a global collaborative study JCO Glob Oncol 6 1428-1438 https://doi.org/10.1200/GO.20.00351 PMID: 32986516 PMCID: 7529504

13. Richards M, Anderson M, and Carter P, et al (2020) The impact of the COVID-19 pandemic on cancer care Nat Cancer 1-3 [http://doi: 10.1038/s43018-020-0074-y] Date accessed:30/06/21 PMID: 32838302 PMCID: 7238956

14. De Guzman R and Malik M (2020) Dual challenge of cancer and COVID-19: impact on health care and socioeconomic systems in Asia pacific JCO Glob Oncol 6 906-912 https://doi.org/10.1200/GO.20.00227 PMID: 32589462 PMCID: 7328111

15. Villain P, Carvalho AL, and Lucas E, et al (2021) IARC COVID-19 Impact Study Group. Cross-sectional survey of the impact of the COVID-19 pandemic on cancer screening programs in selected low- and middle-income countries: study from the IARC COVID-19 impact study group Int J Cancer 149 97-107 https://doi.org/10.1002/ijc.33500 PMID: 33533501 PMCID: 8014228 
16. Viswanathan AN, Beriwal S, and De Los Santos JF, et al (2012) American Brachytherapy Society. American Brachytherapy Society consensus guidelines for locally advanced carcinoma of the cervix Part II: high-dose-rate brachytherapy Brachytherapy 11 47-52 https:// doi.org/10.1016/j.brachy.2011.07.002 PMID: 22265437 PMCID: 3489267

17. Albuquerque K, Hrycushko BA, and Harkenrider MM, et al (2019) Compendium of fractionation choices for gynecologic HDR brachytherapy-An American Brachytherapy Society Task Group Report Brachytherapy 18 429-436 https://doi.org/10.1016/j.brachy.2019.02.008 PMID: 30979631

18. Hendry J, Jones GW, and Mahantshetty UM, et al (2017) Radiobiological analysis of outcomes using external beam radiotherapy plus high dose-rate brachytherapy (4x7 Gy or 2x9 Gy) for cervical cancer in a multi-institution trial Int J Radiat Biol Phys 99 1313-1314 https://doi.org/10.1016/j.jijobp.2017.09.009

19. Mohindra P, Beriwal S, and Kamrava M (2020) Proposed brachytherapy recommendations (practical implementation, indications, and dose fractionation) during COVID-19 pandemic Brachytherapy 19 390-400 https://doi.org/10.1016/j.brachy.2020.04.009 PMID: 32423787 PMCID: 7252026

20. Torre LA, Islami F, and Siegel RL, et al (2017) Global cancer in women: burden and trends Cancer Epidemiol Biomarkers Prev 26 444-457 https://doi.org/10.1158/1055-9965.EPI-16-0858 PMID: 28223433

21. United Nations (2021) The Sustainable Development Goals Report 2020 (United Nations) [https://unstats.un.org/sdgs/report/2020/TheSustainable-Development-Goals-Report-2020.pdf]

22. World Health Organization (2020) Global Strategy to Accelerate the Elimination of Cervical Cancer as a Public Health Problem (Geneva: World Health Organization)

23. Canfell K, Kim JJ, and Brisson M, et al (2020) Mortality impact of achieving WHO cervical cancer elimination targets: a comparative modelling analysis in 78 low-income and lower-middle-income countries Lancet 395 591-603 https://doi.org/10.1016/S01406736(20)30157-4 PMID: 32007142 PMCID: 7043006

24. Yusuf A (2020) Cancer care in the time of COVID-19-a perspective from Pakistan Ecancermedicalscience 141026 PMID: 32346392 PMCID: 7176062

25. Ting FI, Sacdalan DB, and Abarquez HS, et al (2020) Treatment of cancer patients during the COVID-19 pandemic in the Philippines Ecancermedicalscience 141040 PMID: 32565893 PMCID: 7289609

26. World Cancer Initiative (2021) Cancer Preparedness In Asia-pacific Progress Towards Universal Cancer Control [https://worldcancerinitiative. economist.com/pdf/Roche-cancer-preparedness-in-asia/Roche_Cancer_White_paper.pdf]

27. Alkatout I, Biebl M, and Momenimovahed Z, et al (2021) Has COVID-19 affected cancer screening programs? A systematic review Front Oncol 11675038 https://doi.org/10.3389/fonc.2021.675038 PMID: 34079764 PMCID: 8165307

28. Cancino RS, Su Z, and Mesa R, et al (2020) The impact of COVID-19 on cancer screening: challenges and opportunities JMIR Cancer 6 e21697 https://doi.org/10.2196/21697 PMID: 33027039 PMCID: 7599065

29. Sharpless NE (2020) COVID-19 and cancer Science 3681290 https://doi.org/10.1126/science.abd3377 PMID: 32554570

30. Editorial (2021) The Lancet Oncology. COVID-19 and cancer: 1 year on Lancet Oncol 22411 https://doi.org/10.1016/S14702045(21)00148-0

31. Gupta S, Maheshwari A, and Parab P, et al (2018) Neoadjuvant chemotherapy followed by radical surgery versus concomitant chemotherapy and radiotherapy in patients with stage IB2, IIA, or IIB squamous cervical cancer: a randomized controlled trial J Clin Oncol 36 1548-1455 https://doi.org/10.1200/JCO.2017.75.9985 PMID: 29432076 
32. Lombe D, Phiri M, and Msadabwe S (2020) Negative impact of the COVID-19 pandemic on the management of cervical cancer patients in Zambia Ecancermedicalscience 14 ed103 https://doi.org/10.3332/ecancer.2020.ed103 PMID: 32728388 PMCID: 7373645

33. Umutesi G, Shyirambere C, and Bigirimana JB, et al (2021) Cancer care delivery innovations, experiences and challenges during the COVID-19 pandemic: the Rwanda experience J Glob Health 1103067 https://doi.org/10.7189/jogh.11.03067 PMID: 33884189 PMCID: 8053392

34. Portaluri M, Barba MC, and Musio D, et al (2020) Hypofractionation in COVID-19 radiotherapy: a mix of evidence based medicine and of opportunities Radiother Oncol 150 191-194 https://doi.org/10.1016/j.radonc.2020.06.036 PMID: 32621832 PMCID: 7329675

35. Monica and Mishra R (2020) An epidemiological study of cervical and breast screening in India: district-level analysis BMC Womens Health 20225 https://doi.org/10.1186/s12905-020-01083-6 PMID: 33028336 PMCID: 7542863

36. Mathur P, Sathishkumar K, and Chaturvedi M, et al (2020) Cancer statistics, 2020: report from national cancer registry programme, India JCO Glob Oncol 6 1063-1075 https://doi.org/10.1200/GO.20.00122 PMID: 32673076 PMCID: 7392737

37. Gupta N, Chauhan AS, and Prinja S, et al (2021) Impact of COVID-19 on outcomes for patients with cervical cancer in India JCO Glob Oncol 7 716-725 https://doi.org/10.1200/G0.20.00654 PMID: 33999708 PMCID: 8162960 